\title{
ÉLÉMENTS DE CONCEPTUALISATION DU TRAVAIL D'ENSEIGNANT CHERCHEUR : L'ACTIVITÉ SUIVI DE MÉMOIRE EST-ELLE HOMOMORPHE À L'ACTIVITÉ ACCOMPAGNEMENT DE VALIDATION DES ACQUIS DE L'EXPÉRIENCE ?
}

\section{Grégory Munoz, Emmanuel Sylvestre, Évelyne Soulard}

\section{ADRESE/CIRNEF | « Les Sciences de l'éducation - Pour l'Ėre nouvelle »}

2013/2 Vol. 46 | pages 41 à 59

ISSN 0755-9593

ISBN 9782918337157

\section{Article disponible en ligne à l'adresse :}

https://www.cairn.info/revue-les-sciences-de-l-education-pour-l-erenouvelle-2013-2-page-41.htm

Distribution électronique Cairn.info pour ADRESE/CIRNEF.

(C) ADRESE/CIRNEF. Tous droits réservés pour tous pays.

La reproduction ou représentation de cet article, notamment par photocopie, n'est autorisée que dans les limites des conditions générales d'utilisation du site ou, le cas échéant, des conditions générales de la licence souscrite par votre établissement. Toute autre reproduction ou représentation, en tout ou partie, sous quelque forme et de quelque manière que ce soit, est interdite sauf accord préalable et écrit de l'éditeur, en dehors des cas prévus par la législation en vigueur en France. Il est précisé que son stockage dans une base de données est également interdit. 


\section{Éléments de conceptualisation du travail d'enseignant chercheur : L'activité suivi de mémoire est-elle homomorphe à l'activité accompagnement de Validation des Acquis de l'Expérience?}

Grégory Munoz", Emmanuel Sylvestre" et Évelyne Soulard"."

Résumé : L'étude caractérise l'activité d'accompagnement du dossier de validation des acquis de l'expérience de l'enseignant-chercheur à travers une analyse issue du paradigme de la didactique professionnelle (Pastré, 2011). Elle explore les homomorphismes (Vergnaud, 1994) entre la situation d'activité connue de suivi de mémoire de master et cette situation d'activité moins connue. Se pose alors la question de savoir si les concepts en acte (Vergnaud, 1999) construits par l'acteur afin de pouvoir gérer la première situation sont mobilisables dans le cadre de la seconde. L'une et l'autre relèvent-elles d'une même classe de situation plus élargie, qui pourrait être relative à l'accompagnement de réalisation d'un travail universitaire?

$\mathrm{Si}$ les deux situations d'activité semblent a priori parentes, il importe cependant d'en dégager les différences. En effet, ces dernières s'avèrent essentielles pour les acteurs, afin de leur permettre de mieux conceptualiser les spécificités de l'activité d'accompagnement en $V A E$, pour laquelle ils sont peu formés.

Mots-clés : Accompagnement VAE. Suivi de mémoire. Didactique professionnelle. Conceptualisation.

\footnotetext{
* Maître de conférences, Université de Nantes, Centre de Recherche en Éducation de Nantes (CREN - EA 2661).

** Docteur en Sciences de l'Éducation, Conseiller pédagogique au Centre de Soutien à l'Enseignement, Université de Lausanne.

*** Chargée mission formation continue, Faculté de Droit et des Sciences Politiques de Nantes.
} 


\section{Introduction : les enjeux de la validation des acquis de l'expérience à l'université}

Selon Mayen et Métral, «la validation des acquis de l'expérience constitue un espace de travail commun fait de dialogue et de négociation entre deux mondes que l'on estime a priori hétérogènes l'un à l'autre», celui de la formation et celui de travail (Mayen \& Métral, 2008, p. 185). Ne pouvons-nous pas émettre l'hypothèse qu'au sein du dispositif de validation des acquis de l'expérience (VAE), nous serions confrontés à deux «mondes professionnels» tels que Béguin (2007, p. 376) définit le terme, c'est-à-dire en tant que «système d'arrière-plan qui découpe dans le réel certain fait ou événement (alors que d'autres faits resteront muets ou silencieux) et sur lequel on s'appuie pour construire une représentation ou un savoir»? De ce point de vue, le "monde professionnel» correspond aussi à la manière dont l'acteur construit la situation professionnelle (Béguin, 2007, p. 376). En effet, dans le dispositif de validation des acquis de l'expérience (VAE), il apparaît que les professionnels du monde de la formation sont confrontés directement à celui du travail.

Le processus de VAE recouvre de multiples enjeux dans une société de la connaissance qui se propose de multiplier les voies d'accès aux diplômes. En effet, il engage pour l'université une "petite révolution", voire une provocation selon l'expression de Cherqui-Houot (2006, p. 98), pour laquelle elle n'est pas préparée, notamment en ce qui concerne les modalités d'activité des acteurs chargés de la mettre en œuvre. Cherqui-Houot (2006, p. 101) a observé «chez les enseignants universitaires (accompagnateurs ou membres du jury) une tendance forte à ramener les caractéristiques des candidats à la VAE à des «standards» étudiants davantage connus d'eux". Nous nous intéressons à une telle tendance. Comme il est nécessaire de "se référer à du connu pour aborder l'inconnu", un des éléments d'une possible formation au processus de suivi VAE pourrait consister en un jeu de mise en correspondance entre ce qui relève de l'activité habituelle et de cette nouvelle activité. En nous appuyant spécifiquement sur la théorie de la conceptualisation de l'action de Vergnaud (1996 et 2007), et de manière plus large à la didactique professionnelle (Pastré, 2011), nous centrons notre propos sur le rôle de l'enseignant chercheur en tant qu'accompagnateur dit référent diplôme ${ }^{1}$, au sein du processus de VAE. Nous considérons comment cet

1. C’est-à-dire référent du diplôme auquel les candidats veulent présenter leur VAE. 
acteur conceptualise sa nouvelle fonction au regard d'une ancienne qu'il maittrise, pour savoir sur quoi il peut appuyer cette nouvelle pratique. Notre problématique concerne plus précisément la question suivante : l'activité d'accompagnement de réalisation de mémoire de master, connue du point de vue de l'acteur, est-elle transposable dans le cadre de l'activité d'accompagnement en VAE, encore inconnue de l'acteur? Et si oui, il s'agit alors de considérer dans quelle mesure et à quelles conditions? Si non, de comprendre pourquoi. Car si effectivement, ces activités peuvent sembler relever de compétences estimées mobilisables chez certains acteurs, nous verrons qu'il peut s'agir en partie d'une sorte de «leurre épistémologique». Celui-ci constitue non seulement une sorte d'obstacle épistémologique bachelardien pour le sujet, mais aussi une contre-intuition quasi-institutionnelle ${ }^{2}$.

Nous exposerons dans la partie suivante, les cadres théorique et méthodologique de notre approche qui s'appuient sur le paradigme de la didactique professionnelle. Cette dernière s'intéresse au développement des adultes et à la manière dont ils construisent des connaissances dans l'action. Au sein de la troisième partie, nous présenterons nos principaux résultats, en précisant, en amont, le contexte de l'étude. Ils concernent les modalités de mise en correspondance entre les deux types d'activité. Enfin, nous déploierons une discussion-conclusion liée à la question de l'activité des enseignants chercheurs, et à des pistes de formation à cette nouvelle fonction d'accompagnement en VAE.

\section{Cadre théorique et méthodologique : de l'identification de l'articulation situation/schème}

\subsection{Champ théorique de référence : la didactique professionnelle}

Notre questionnement est étayé par l'approche issue de la didactique professionnelle (Pastré, Mayen \& Vergnaud, 2006; Pastré, 2011), qui s’intéresse à l'analyse cognitive des acteurs en situations de travail, en vue de constituer des situations de formation basées sur les "organisateurs de l'activité» mis au jour avec l'aide des travailleurs. L'idée principale est d' «apprendre des situations», en considérant le caractère problématique, et par conséquent potentiellement formatif de

2. Ce qui se joue également dans le cadre de la constitution d'un référentiel d'activités d'enseignant chercheur. 
ces dernières. Samurçay et Pastré $(2004$, p. 3) précisent que la didactique professionnelle est née de la rencontre d'un champ de pratiques, la formation professionnelle continue, et de deux champs théoriques, l'ergonomie cognitive (Leplat, 1997), et la didactique des disciplines. Cette dernière a transmis une double préoccupation épistémologique et développementale à partir de la théorie de la conceptualisation dans l'action (Vergnaud, 1996). Dans cette théorie, nous nous référons plus précisément à la notion de schème, en tant qu' "organisation invariante de l'activité pour une classe de situations donnée", liée à la prise en compte des formes organisées de l'action coordonnée issue de l'œuvre de Piaget (1967), qui considère le développement comme une adaptation. Cette approche montre une continuité de la connaissance entre ses différentes formes (Vergnaud, 1999) :

- depuis sa forme «opératoire» ou "agie» : c'est-à-dire celle qui est mise en acte et qui est relative aux schèmes (essentiellement sensori-moteurs chez le bébé, puis d'ordre symboliques et conceptuels, par la suite); elle reste relativement implicite et elle est plus classiquement définie en tant que "compétence»;

- vers sa forme "prédicative» ou "énoncée» : celle-ci correspond à celle qui est mise en mots, formalisée, et par conséquent souvent explicitée et consciente;

- voire vers une forme "théorisée»: relative à celle, qui parmi la forme prédicative, obtient un autre statut, en ce qu'elle est axiomatisée, c'est-à-dire logicomathématiquement établie et relève d'une approche nomothétique (Piaget, 1950), c'est-à-dire aspirant à des lois génériques.

Cette dernière forme est liée à un apport complémentaire (Munoz, 2007).

De ces perspectives, il apparaît possible de dégager une «implication didactique», énonçable de la manière suivante: si, comme le soutient Vergnaud (1996), "au fond de l'action, la conceptualisation», celle-ci restant pertinente en situation mais aussi implicite, alors on peut "apprendre des situations" (Pastré, 1999 (a)). Autrement dit, il s'agit d'une «implication didactique» énonçable à partir des situations de travail, selon la didactique professionnelle et non plus seulement à partir des savoirs, selon les approches des didactiques des disciplines scientifiques et techniques, même si ces derniers sont également mis en scènes en situation, par exemple à partir de la théorie des situations didactiques (Brousseau, 1998).

Mais à quelles conditions peut-on apprendre des situations? Pastré (1999 (a)) envisage la part cognitive de l'activité, liée au fait de pouvoir établir un diagnostic de la situation professionnelle, en vue d'agir pertinemment, en considérant l'ensemble des variables en jeu. Cependant, cet accès ne va pas de soi, d'où 
l'importance de remonter aux formes prédicatives, en les articulant aux formes opératoires, c'est-à-dire en lien avec les situations de travail. Ce qui permet une certaine prise de recul vis-à-vis de ces situations, par une analyse qui ne fait ni l'économie du travail réel, ni celle de l'articulation aux savoirs théoriques, qui devront être contextualisés en situation (Pastré, 2011) : c'est-à-dire en recourant à l'analyse approfondie des situations, notamment à travers l'analyse de l'activité chère à l'ergonomie (Leplat, 1997). N'est-ce pas d'ailleurs en partie ce que propose le processus de VAE? Cependant, c'est en partie seulement, dans la mesure où l'on peut parfois assister au fait d'éviter l'analyse d'une situation spécifique pour en rester à un niveau générique d'énonciation, sans prendre en compte les variables contextuelles ni l'articulation aux savoirs scientifiques et techniques du diplôme.

D'autant que Pastré (1997, p. 90) distingue trois types d'apprentissage: l'apprentissage avant lié à l'activité d'appropriation des "connaissances opérationnelles" nécessaires pour maîtriser la situation de référence; l'apprentissage pendant: par l'exercice de l'activité ou «sur le tas"; et l'apprentissage après: par l'analyse de l'activité. La didactique professionnelle met l'accent sur ce dernier type pour dépasser la combinaison des deux autres. «Refaire, reproduire, imiter sont certes des moyens d'apprentissage, mais le plus puissant semble bien être celui qui consiste à reconstruire " énonce d'ailleurs Pastré (1999 (b), p. 31). Nous adoptons cette troisième voie en re-contruisant en partie ${ }^{3}$ l'expérience éprouvée.

\subsection{Origine du présent questionnement : d'une intuition première déstabilisée}

Ce questionnement prend sa source à partir d'une première intuition issue d'une pratique personnelle ${ }^{4}$ mais également collective (Dumas, Munoz \& Parage, 2010), en tant qu'accompagnateur référent, c'est-à-dire référent du diplôme brigué par le candidat à la VAE. Pour faire face à cette situation, relativement

3. Cette re-construction constitue une forme d'analyse de pratique "après coup", qui ne bénéficie cependant pas directement des traces de l'activité, contrairement aux approches plus «classiques" de la didactique professionnelle.

4. S'il est stimulant d'avoir accepté une nouvelle activité pour laquelle on peut spontanément s'identifier comme légitime, au regard de la requête émise, il peut être décevant d'avoir à constater, par la suite, que celle-ci est due à une pénurie de volontaires pour accompagner la VAE, étant donné le peu de décharges horaires qu’elle génère. 
nouvelle pour notre département, mais également pour l'université $q u i$ mettait en place son service de VAE, nous avons eu à mobiliser une part connue ${ }^{6}$ de notre activité. Pour cela, nous avons eu recours à ce que nous avons été amenés à construire quand nous intervenions en tant que responsables de suivi de mémoire de master. Ce recours est-il pertinent? Cette contre-intuition, qui nous est apparue a posteriori (en tension par rapport à nos a priori plutôt favorables à ce recours), pourrait s'énoncer comme suit : avoir recours à un schème, en tant qu' "organisation invariante de la conduite pour une classe donnée de situations " (Vergnaud, 1996, p. 283), constitué chez l'enseignant-chercheur, et déployé dans le cadre du suivi de mémoire de master, constitue-t-il une aide ou un obstacle à l'activité de suivi en VAE? Dans le cadre du paradigme de la théorie des champs conceptuels (Vergnaud, 1990), il s'agirait de se demander si ces deux activités relèvent de la même "classe de situations».

\subsection{Vers un re-questionnement dans le champ de la théorie des champs conceptuels (TCC)}

Il s'agit de faire appel au même champ conceptuel, en entendant par concept, les deux formes de la connaissance prédicative mais également opératoire, implicite pour l'acteur. Précisons toutefois quelques éléments importants de cette approche. Selon la théorie des champs conceptuels, «un champ conceptuel est un ensemble relativement large de situations, d'invariants et de signifiants, dans lesquels plusieurs concepts de nature différente sont en interaction, avec plusieurs compétences, plusieurs systèmes symboliques» (Vergnaud, 1987, p. 841). C'est pourquoi, Vergnaud, par ailleurs, précise que : «du fait que le concept se réfère à plusieurs situations, qui elles-mêmes ne se laissent pas analyser à l'aide d'un seul concept, il nous faut étudier des champs conceptuels, c'est-à-dire des ensembles de situations et de réseaux organisés de concepts qui se forment et se développent conjointement et en étroite interaction» (Vergnaud, 1995, p. 184).

5. Si de 2002 à 2010, la part de la VAE dans les établissements d'enseignement supérieur est en augmentation, puisqu'elle est passée de 1140 à 4064 (MEN, 2011, p. 1), en 2010, l'université en question ne présentait qu'une vingtaine de dossiers selon l'estimation de la DEPP $(2011$, p. 2).

6. Terme à nuancer du fait qu'il s'agit davantage d'une connaissance en acte déployée en situation que d'un savoir explicite. 


\subsection{Méthodologie : des limites de l'introspection rétrospective vers la comparaison de morphisme}

À l'aune de cette question et de la littérature sur l'activité liée au processus de VAE (Mayen, 2004 et 2008), nous explorons l'activité d'accompagnateur référent-diplôme dans le cadre d'un master professionnel particulier. Nous avons souhaité dépasser les limites de l'introspection rétrospective, même si nous avons tenu à explorer le point de vue de l'activité cognitive des acteurs en eux-mêmes. Dans cette perspective, notre méthodologie d'analyse a consisté en deux temps. D'une part, elle a considéré l'expérience de cette pratique en tant que matière à travailler, à la manière des candidats à la VAE, mais sans démarche spécifique préalable. D'autre part, elle a cherché à revenir sur cette pratique, liée à l'accompagnement par trois enseignants chercheurs référents du diplôme pour chacun des deux candidats (d'une même institution et pour briguer le même diplôme), à partir des éléments de la théorie de la conceptualisation dans l'action. À cet égard, nous procédons à une analyse plus systématique de recherche des différences et des invariants entre les deux situations, en vue d'estimer leur "morphisme». Des éléments pourraient être définis comme invariants en ce qu'ils relèvent d'un "homomorphisme» entre les deux structures d'activité, ou à l'inverse comme éléments de différence. La question principale pourrait être posée ainsi : les deux situations sont-elles suffisamment «homomorphes» pour laisser à penser qu'elles relèvent d'une même «classe de situations »?

La notion d'homomorphisme ${ }^{7}$ (Vergnaud, 1994) soutient l'existence d'un morphisme, c'est-à-dire d'une structure de forme (Simondon, 1958/1989), entre réel et représentation conceptuelle, entre référents et référés, ou pour être encore plus précis, entre éléments cognitifs opératoires du concept (les signifiés) et éléments symboliques de ces derniers (les signifiants), eux-mêmes organisés en systèmes. Il s'agirait non pas de structures isomorphes (c'est-à-dire liées à des éléments "égaux" de part et d'autre) mais de structures "homomorphes» qui relèvent de "correspondances entre les éléments de l'ensemble d'arrivée et des classes d'éléments de l'ensemble de départ» (Vergnaud, 1994, p. 27).

7. Rappelons que Vergnaud est à l'origine didacticien des mathématiques; ce qui explique le recours à une telle terminologie. 


\section{3. Éléments d'analyse : de quelques obstacles à considérer ces deux activités comme appartenant à une même classe de situations}

Avant de présenter les résultats des deux types d'analyses menées, intuitive, d'une part, ou plus systématique par comparaison de morphisme, d'autre part, il convient d'établir le contexte de cette étude.

\subsection{Contexte : un master lié à l'analyse réflexive des situations de travail}

Le diplôme pour lequel les candidats ont été suivis par l'accompagnateur dont les éléments de conceptualisation sont étudiés, est le master 2 «Métiers, pratiques et recherches en éducation et formation" (MPREF), parcours professionnel «Formation de formateur par l'analyse des situations de travail» (FFAST). Cette formation a la particularité de préparer des étudiants et professionnels en reprise d'étude à «devenir des formateurs en concevant des situations de formation à partir de l'analyse des situations professionnelles" (justement dans le paradigme de la didactique professionnelle). Les débouchés proposés sont relatifs aux fonctions de conseiller pédagogique, consultant en entreprise, formateur d'adulte ou en insertion, ou même accompagnateur de VAE. Une part de la présentation de ce titre précise qu' «il s'agit de dépasser une logique de «reproduction» des professions, pour penser et théoriser leurs évolutions dans la société par des apports théoriques, méthodologiques ainsi que par la pratique d'une posture réflexive». Cette formation s'adresse d'abord aux enseignants et formateurs souhaitant devenir formateurs de formateurs mais aussi aux professionnels souhaitant devenir formateurs. C'est pourquoi, dans un certain nombre de cas, le recours à la VAE peut s'avérer intéressant.

Ce master comporte des savoirs d'ordre technique liés aux activités visées qui sont présentées dans la fiche $\mathrm{RNCP}^{8}$, dont voici un extrait :

8. Pour de plus amples détails sur la fiche RNCP, voir à la fin du Cahier pédagogique du diplôme, en ligne : [http://www.univ-nantes.fr/adminsite/objetspartages/liste_fichiergw.jsp?OBJET=DOCUMENT $\& \mathrm{CODE}=1288252960087 \& \mathrm{LANGUE}=0$; pour de plus amples détails sur cette formation, voir la fiche diplôme en ligne : http://www.univ-nantes.fr/M2FFAST/0/fiche formation/]. 
- Il conçoit, met en œuvre et évalue des actions de formation en référence à des situations professionnelles réelles situées dans des organisations de travail singulières;

- Il contribue, par l'accompagnement des individus, des collectifs de travail et des organisations, à la formalisation des savoirs d'action et d'expérience pour permettre la transmission des connaissances incorporées et/ou la validation des acquis;

- Il met en relation les acquis des personnes et les modes de pilotage et de gestion des organisations (Gestion Prévisionnelle des Emplois et Compétences, processus qualité, Droit Individuel de Formation, ....).

\section{Tableau 1 : Extrait de la fiche RNCP du Master FFAST présentant synthétiquement l'activité visée}

La conception et l'évaluation d'actions de formation de ce master professionnel ainsi que l'accompagnement et la formalisation des savoirs se réalisent dans le cadre du paradigme de la didactique professionnelle. Il se déploie selon une forme déontologique inspirée de l'ergonomie, c'est-à-dire dans le sens d'une collaboration des acteurs de terrain qui prennent part à l'analyse de leur propre activité, en vue de leur développement professionnel. L'énoncé des compétences attestées renvoie à quatre grands thèmes de mission : a) - expertiser les modèles de formation, b) - analyser des situations de travail, c) - analyser les effets identitaires des situations de travail et de formation, d) - articuler recherche et développement professionnel. Concernant la VAE, il s'agit d'une validation partielle en ce sens où n'est pas admise la possibilité de valider la partie mémoire de recherche en VAE. L'argument tenu est que ce master est lié à une formation par la recherche, compte tenu des spécificités avancées.

\subsection{Première analyse « intuitive» à partir d'un retour sur l'activité}

Cette section, par le recours à un retour sur l'activité menée d'accompagnateur-référent du diplôme, se propose de présenter le mouvement déployé depuis une intuition a priori vers une contre-intuition a posteriori. L'intuition a priori était liée à une croyance en des ressemblances entre les deux situations d'activités. La contre-intuition a posteriori a été liée à la prise en compte progressive des différences, conceptualisées à la suite de l'expérience de suivi de VAE.

Dans le cadre d'un retour à la théorie de la conceptualisation dans l'action, il s'agirait de considérer que face à la nécessité de s'adapter à une situation nouvelle, 
tout acteur se réfere à ses ressources constituées : des ressources internes de l'ordre des schèmes et concepts construits au cours de l'expérience (Piaget, 1967; Pastré, 2011); mais également des ressources externes, sociales, car relatives au recours aux pairs ou au collectif ou encore culturelles, langagières ou instrumentales (le recours à l'explicitation ou aux documents par exemple), en références aux apports de la théorie de la médiation (Vygotski, 1934; Mayen, 1999; Rabardel, 2005).

Dans le cas de la VAE, l'acteur enseignant-chercheur se réfère à ses ressources : 1) internes, ses schèmes d'action construits dans le cadre d'activité de suivi de mémoire de master, mais aussi des ressources externes; 2) sociales, avec le recours aux pairs : le groupe lié à l'équipe pédagogique du diplôme, les membres du pôle VAE; 3) culturelles, langagières ou instrumentales : le recours aux documents de référence donnés par le pôle VAE, à la fiche RNCP ou encore à la grille de critères d'évaluation attendus pour chaque UE conçue de manière ad hoc par l'équipe pédagogique du diplôme, dont font partie les conseillers VAE référents diplôme et y compris l'acteur dont il est question ici. Dans le cadre de notre propos, nous nous centrons davantage sur les ressources internes liées aux schèmes d'action du sujet.

En ce qui concerne les ressemblances a priori entre les deux situations d'activités, vues du point de vue de l'acteur qui les a expérimentées, plusieurs sont à mettre en avant. La première ressemblance concerne une activité d'accompagnement. La seconde est relative au fait qu'il s'agit d'une relation d'accompagnement avec un étudiant, nécessitant, dans les deux cas, la production d'un écrit universitaire. Cet écrit s'inscrit dans une recherche de réponse à des standards académiques attendus, par exemple: construire une problématique à partir d'une lecture de la littérature d'un domaine scientifique et recueillir des données empiriques en les analysant à partir d'un paradigme théorique et méthodologique, en vue de répondre à une question de départ problématisée au sein de ce paradigme.

Si en début d'activité, l'accompagnateur avait pensé pouvoir recourir à son organisation de suivi de mémoire de master 1 et 2, en vue de la transposer en accompagnement VAE, il est apparu, au cours de l'avancement, de plus en plus de différences entre les deux activités.

En ce qui concerne les différences a posteriori entre les deux situations d'activités, une série de nuances peut être émise :

- Il ne s'agit pas tout à fait d'une relation avec un étudiant car le candidat VAE n'a pas les mêmes caractéristiques, voire un statut différent (pouvant rendre 
problématique l'accès à une carte d'étudiant pour bénéficier de la bibliothèque universitaire par exemple).

- Il ne s'agit pas non plus exactement d'un même type de production universitaire, puisque celle-ci cherche à répondre à d'autres attendus, notamment au fait d'être capable de partir de l'expérience pour la ré-interpréter, dans le but de la référer aux éléments du référentiel d'un diplôme. En outre, il ne contient pas exactement les mêmes rubriques, ou peut même en contenir des spécifiques, telles que le tableau de correspondances. Celui-ci présente les liens entre les éléments du référentiel du diplôme brigué et les éléments d'expérience et de formation du candidat, que ce dernier estime intéressants à présenter aux membres du jury.

\subsection{Seconde analyse par comparaison de morphisme situationnel}

Il s'agit dans ce second cas de proposer une analyse par comparaison de morphismes situationnels, c'est-à-dire par comparaison plus systématique entre les deux structures des situations en question, notamment en prenant appui sur la littérature issue des travaux de la didactique professionnelle relatifs à la VAE. Cette analyse permet de dégager ce qui correspond à la "compétence critique" (Vergnaud, 1995 (b)) dans l'activité d'accompagnateur de VAE, en ce sens où cela est apparu contre-intuitif a posteriori d'avoir considéré comme intuitivement comparables les deux situations; et ceci d'autant que cette activité peut difficilement s'apprendre uniquement par l'expérience (Mayen, 2004). Cela entraîne un déplacement vers un point de vue plus analytique, en examinant plus systématiquement les correspondances entre les deux situations d'activités, dont nous pointons ici quelques éléments?

En ce qui concerne les invariants entre les deux types de situation, l'acteur peut se référer à son expérience de la première pour orienter son activité vers la seconde. En effet, elles relèvent toutes deux d'une forme d'accompagnement au sein d'une culture scientifique d'une part et dans l'institution universitaire d'autre part. Cela peut toutefois être lié à une différence de degré, pouvant apparaître plus important dans le cas de la VAE. Par exemple, construire une problématique à partir d'une lecture de la littérature d'un domaine, dans le

9. En effet, une analyse de l'activité permettant de repérer plus directement la structure de l'action de l'une et l'autre situation reste à mener. 
cas de la VAE, nécessite aussi de se référer au référentiel du diplôme. Recueillir des données, dans le cadre de la VAE, concerne essentiellement celles issues de l'expérience du candidat.

Relativement aux premiers éléments de différences repérés, nous pouvons énoncer que la VAE constitue une activité éminemment collective, nécessitant un co-suivi inter-services, dans un contexte institutionnel particulier, souligné aussi par Mayen (2004). En effet, ce caractère collectif est intrinsèque à la VAE, alors qu'en suivi de mémoire, il n'intervient qu'en cas de problème. Voici une anecdote issue de l'accompagnement en VAE : le délai d'attente de réponse au candidat sollicitant le retour des accompagnateurs à l'égard d'une production à examiner a pu considérablement différer. En effet, le délai s'avère court (de l'ordre d'un ou deux jours) du côté de l'accompagnateur référent-méthode du pôle VAE, un service central de l'université spécifiquement dédié à cette activité d'ordre méthodologique, et qui examine plutôt la forme de la production. À l'opposé, le délai du côté du conseiller référent-diplôme, dont le retour relève $\mathrm{du}$ fond de la production écrite du candidat, s'avère nettement plus long (de l'ordre d'une ou deux semaines). Il est à noter que l'accompagnement VAE relève de l'activité principale du premier et d'une activité annexe pour le second.

La VAE constitue également une activité auprès d'un candidat qui ne suit pas d'enseignements comme un étudiant. Dans cette perspective, l'enseignant accompagnateur référent doit adopter une position $\mathrm{d}^{\text {'«incarnation } \mathrm{du}}$ référentiel» plus importante que dans le cas d'un suivi de mémoire de master. En effet, en VAE, le diplôme dans son ensemble se voit "cristallisé» en une unique personne. Cette dernière peut dès lors se sentir moins légitime, car elle ne maitrise pas l'ensemble des connaissances du diplôme, contrairement à la situation suivie de mémoire, où elle co-construit avec l'étudiant son sujet, en l'orientant davantage vers ce qu'elle maîtrise. Qu'en est-il en VAE des unités d'enseignement que l'accompagnateur ne connait pas? En effet, si le candidat n'a pas suivi d'enseignements comme un étudiant, en revanche, il cherche à en bénéficier indirectement : soit lors des rencontres avec l'accompagnateur, soit au moment de la soutenance devant le jury, qu'il souhaite également formative. Par exemple, il peut accéder à des concepts, via des concepts potentiels, comme le montre une étude de Kostulski et Prot (2004). Ces auteurs mettent en évidence le fait que "l'ajustement mutuel dans l'interlocution constitue un lieu et un facteur de développement de ces activités conversationnelles» (p. 438), puisque 
le membre du jury expérimenté cherchera à orienter le candidat vers une explicitation de son expérience en référence aux savoirs du diplôme ${ }^{10}$.

Dans une étude visant à caractériser l'accompagnement en VAE, Mayen (2004) fait référence au "malaise résiduel des accompagnateurs", notamment face aux «difficultés des institutions à définir le travail à accomplir et à organiser un cadre structurant». Ceux-ci ont besoin de se situer par rapport à leur propre position, avec la hantise de mal préparer les candidats; ce qui par conséquence "surévalue» l'image de ce que devrait faire un accompagnateur de VAE (Mayen, 2004, p. 9). Il note également comment un accompagnateur «trop aidant» déstabilise l'exigence d'équité entre candidats (p. 10), ou au contraire comment un accompagnateur peut s'autoriser à résister aux "demandes abusives" des candidats. Ce qui montre l'importance de conceptualiser le cadre prescriptif et réglementaire souvent sous-estimé (p. 11). L'accompagnement en VAE relève «d'un processus d'élaboration qui demande beaucoup plus que de l'information ou de l'expérience» (Mayen, 2004, p. 21). L'assimiler à un dispositif de formation constitue un obstacle à la définition de la fonction, qui nécessite «l'invention de forme d'action par les accompagnateurs" (Mayen, 2004, p. 13). Il relève aussi d'une activité de service, où l'usager comme le professionnel ont à apprendre de la «situation qui est cadrée à la fois par le processus global de VAE et à la fois par la transaction plus limitée d'accompagnement» (p. 16), en fonction de la répartition des tâches entre candidat et accompagnateur et la négociation du contrat d'accompagnement (p. 19).

\section{Discussion et conclusion : apports et limites}

À partir d'une analyse comparée, avec le recours à la notion d'homomorphisme adaptée des travaux de Vergnaud (1994), nous avons cherché à comprendre comment procède un acteur devant une nouvelle activité. Cette forme d'approche cherche à compléter celles liées aux postures d'accompagnement, conduire, guider ou escorter (Paul, 2009), que peuvent adopter les acteurs, en considérant l'activité cognitive de ces derniers dans le cadre de la théorie de la conceptualisation dans l'action (Vergnaud, 1996 et 2007). Nous avons pu constater que les conceptualisations sont référées à «du connu pour aborder l'inconnu»; mais qu'il ne s'agit toutefois pas de la même «classe de situations». Mayen (2004,

10. En «disloquant la situation concrète» au regard des propriétés du concept (p. 439). 
p. 12) parle à cet égard d'une fonction "assimilée ou supposée proche». "Le jury est supposé porteur des attentes sociales formalisées dans les documents de références (fiches RNCP et référentiels). De ce point de vue, le dossier et l'entretien avec le jury sont, a priori, des produits résultant d'une activité formatée, au sens où elle doit correspondre à des formats. On est très loin de l'expression libre d'une expérience subjective» (Mayen, 2004, p. 19). Mayen resitue la place de l'institutionnel dans la situation de travail. En effet, l'entrée dans la culture VAE pour un nouvel acteur nécessite, justement, la conceptualisation de ces spécificités, par exemple liées aux exigences d'obtention d'un titre ou à l'organisation des ressources pour y parvenir (Mayen, 2004, p. 18). Par conséquent, les accompagnateurs ${ }^{11}$ pourraient bénéficier d'une formation, pouvant les amener à comprendre davantage les spécificités de l'activité de suivi en VAE, par exemple en distinguant mieux les deux situations comme nous l'avons esquissé ici.

Le processus mis au jour semble procéder d'une forme d'équilibration entre activité de l'acteur et "réponse" de la situation, renvoyant à l'articulation situation-schème chère à Vergnaud. Pour dépasser cette perspective, nous pourrions en nous inspirant de Simondon (1958/1989), considérer le processus cognitif de transformation de la situation par le sujet, partant du fait que la situation est constituée par le sujet lui-même en fonction de ses intentions d'action. Dans la dynamique de ce processus, l'on passerait d'une situation où l'organisation de l'action est plus ou moins stabilisée, métastable dirait Simondon, (1958/1989) à la situation qui en diffère, transformant en retour le sujet, «l'individuation» s'instanciant également à partir du potentiel du «milieu». Et comme celui-ci diffère, il impose un changement d'organisation de l'action de la part de l'acteur; ce dernier pouvant prendre en compte cette différence progressivement "perçue», parce que progressivement "conceptualisée». Le propos tenu ici cherchait notamment à mettre en exergue cette nouvelle activité afin de mieux la distinguer d'avec des activités connexes, dont il s'agit de montrer qu'elles sont loin d'être totalement et symétriquement homomorphes. Mieux reconnaître la spécificité de cette activité, c'est se donner les moyens de mieux la caractériser, d'en comprendre les tenants et les aboutissants. C'est justement dans la considération de nouveaux écarts au sein de l'activité que peuvent advenir des «situations potentielles de développement» (Mayen, 1999). Cependant, la mise en

11. Dans notre cas, mais il s'agirait aussi des membres du jury selon Cherqui-Houot (2006, p. 101). 
avant des différences ne risquerait-elle pas paradoxalement de dissuader encore plus $^{12}$ les quelques enseignants-chercheurs volontaires à l'accompagnement en VAE? Comment faire pour y remédier?

Au-delà de cette première analyse, un intérêt davantage porté à l'activité telle qu'elle est déployée par un ensemble d'accompagnateurs donne à voir que ces derniers sont amenés à recourir à un ensemble de ressources autres que leurs propres ressources internes, et notamment des ressources qu'ils sont parfois amenés à concevoir eux-mêmes. Une étude plus systématique des morphismes entre les deux situations reste à mener. Ce travail pourrait se constituer dans le cadre d'une formation-action avec un collectif d'acteurs en situation, formés aux concepts et méthodes de la didactique professionnelle, pour en faire des «agents didactiques» capables, à l'instar des "agents ergonomiques» dans la cadre de recherche-action en analyse du travail (Guérin et al, 1991), de réflexivité en vue d'améliorer, par son explicitation, la conceptualisation de leur activité.

\section{Bibliographie}

BÉGUIN P. Innovation et cadre sociocognitif des interactions concepteurs-opérateurs : une approche développementale. Le travail humain, 2007, vol. 70, n 4 , pp. 369-390.

Brousseau G. Théorie des situations didactiques. Grenoble : La Pensée Sauvage, 1998.

Cherqui-Houdot I. VAE : les universités à l'épreuve de l'expérience. Savoirs, 2010, n' 10 , pp. 95-112.

Dumas M., Munoz G. \& Parage P. Le travail de/et sur l'expérience à l'occasion d'un processus de validation des acquis de l'expérience (VAE) : quand l'activité productive porte sur la présentation de l'expérience orientée par un référentiel de certification-, comment saisir, qualifier, accompagner ce qui se joue du côté de l'activité constructive du sujet? Table ronde. Journée d'études du Master 2 professionnel FFAST (formation de formateurs à l'analyse des

12. Tel est déjà le cas, compte tenu de la charge d'activité complémentaire peu reconnue de surcroît, comprenant 5 heures par participation à un suivi de VAE, dont 1 heure d'entretien d'expertise en amont, 3 heures d'accompagnement et 1 heure de jury en aval. 
situations de travail) La circulation des savoirs entre recherche et formation, 14 juin 2010, Nantes.

Guérin F., Laville A., Daniellou F., Duraffourg J. \& Kerguelen A. Comprendre le travail pour le transformer: la pratique de l'ergonomie. Montrouge : Édition Anact, 1991.

Kostulski K. \& Рвот B. L'activité conversationnelle d'un jury de validation d'acquis : analyse interlocutoire de la formation d'un concept potentiel. Psychologie française, 2004, $\mathrm{n}^{\circ}$ 49, pp. 425-441.

LEPLAT J. Regard sur l'activité en situation de travail - Contribution à la psychologie ergonomique. Paris : PUF, 1997.

MaYen P. Caractériser l'accompagnement en VAE : une contribution de la didactique professionnelle. Education permanente, 2004, n 159 , pp. 7-22.

MaYen P. L'expérience dans les activités de Validation des Acquis de l'Expérience. Travail et Apprentissages, 2008, $\mathrm{n}^{\circ} 1$, pp. 58-75.

MaYen P. \& MÉtral J.-F. Compétences et validation des acquis de l'expérience. Formation Emploi, 2008, $\mathrm{n}^{\circ} 101$, pp. 183-197.

Ministère de l'Education Nationale. La validation des acquis de l'expérience (VAE) dans les établissements d'enseignement supérieur en 2010. Note d'information, DEPP, 2011. Document consultable sur : [http://media.education.gouv.fr/ file/2011/56/4/DEPP-NI-2011-30-validation-acquis-experience-etablissements-enseignement-superieur-2010_203564.pdf].

Munoz G. L'analyse de quelques «mouvements cognitifs» entre les différentes formes de la connaissance : repères pour la formation. Recherches en Éducation, 2007, $\mathrm{n}^{\circ} 4$, pp. 39-50.

Pastré P. Didactique professionnelle et développement. Psychologie française, 1997, vol. 1, n 42, pp. 89-100.

Pastré P. Apprendre des situations. Éducation Permanente, 1999 (a), n 139.

Pastré P. La conceptualisation dans l'action : bilan et nouvelles perspectives. Éducation permanente, 1999 (b), n 111, pp. 13-35.

PAstré P. La didactique professionnelle: approche anthropologique du développement chez les adultes. Paris : PUF, 2011.

Pastré P., Mayen P. \& Vergnaud G. La didactique professionnelle. Revue française de pédagogie, 2006, $\mathrm{n}^{\circ} 154$, pp. 145-198.

Piaget J. Logique et connaissance scientifique. Paris : Gallimard, 1967. 
Paul M. L'accompagnement : d'un dispositif social à une pratique relationnelle spécifique. In : Barbier J.-M., Bourgeois E., Chapelle G. \& Ruano-Borbalan J.-C. (Dir.). Encyclopédie de la formation. Paris : PUF, 2007, pp. 613-646.

Rabardel P. Instrument, activité et développement du pouvoir d'agir. In : Lorino P. \& TEulier R. (Éd.). Entre connaissance et organisation: l'activité collective. Paris : La Découverte, 2005, pp. 251-265.

SAmurçAY R. \& Pastré P. (Éd.). Recherches en didactique professionnelle. Toulouse : Octarès, 2004.

Simondon G. Du mode d'existence des objets techniques. Paris : Aubier, 1989

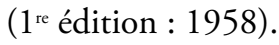

Vergnaud G. La fonction de l'action et de symbolisation dans la formation des connaissances. In : Piaget J., Mounoud P. \& Bronckard J.-P. (Éd.). Psychologie. La Pléiade, XLVI. Paris : Gallimard, 1987, pp. 821-843.

Vergnaud G. La théorie des champs conceptuels». Recherche en Didactique des Mathématiques, 1990, $\mathrm{n}^{\circ} 10(2.3)$, pp. 133-170.

Vergnaud G. Homomorphismes réel-représentation et signifié-signifiant. Exemple en mathématiques. Didaskalia, 1994, n 5, pp. 25-34.

Vergnaud G. Au fond de l'apprentissage, la conceptualisation. Actes de la VIII École d'été de didactique des mathématiques, 1995, pp. 174-185.

Vergnaud G. Évolution du travail et formation des compétences. Le Monde du mercredi 20 décembre 1995.

Vergnaud G. Au fond de l'action, la conceptualisation. In : Barbier J.-M. (Éd.). Savoirs théoriques et savoirs d'action. Paris : PUF, 1996.

VERGNAUd G. La forme opératoire de la connaissance : un beau sujet de recherche fondamentale et appliquée. In : Club CRIN (Éd.). Entreprises et compétences, le sens des évolutions. Paris : Ecrin, 1999, pp. 193-202.

Vergnaud G. Représentation et activité : deux concepts associés. Recherches en éducation, 2007, n 4, pp. 9-22.

Vygotski L. S. Pensée et langage. Paris : Messidor, 1985. 


\title{
A contribution to conceptualizing academic work: is the supervision of a dissertation homomorphous with the supervision of Accreditation of Prior and Experiential Learning?
}

\begin{abstract}
This study seeks to establish the characteristics of the supervision by academics of the process of Accreditation of Prior and Experiential Learning (APEL) using an approach stemming from the paradigm of professional didactics (Pastré, 2011). It explores the homomorphisms (Vergnaud, 1994) between the familiar situation of supervising a Master's dissertation and this less familiar situation. At this point, the question is raised whether the concepts in act (Vergnaud, 1999) created by academics to deal with the first situation can be transferred to the second. Do they both belong to a wider category of situations which would characterize the supervision of academic work?

Although these two activities seem at first sight to be related, it is important to point out their differences. Indeed, these differences turn out to be essential for academics in order for them to better conceptualize the specificity of the supervision of the APEL process in which they have received little training.
\end{abstract}

Keywords: APEL supervision. Master's dissertation supervision. Professional didactics. Conceptualization. 


\section{Elementos de conceptualización del trabajo del docente-investigador : ¿La actividad de seguimiento de una memoria es homomorfa a la actividad de acompañamiento de la Validación de competencias Adquiridas mediante la Experiencia laboral (VAE)?}

Resumen : El estudio caracteriza la actividad de acompañamiento del expediente de la acreditación de la experiencia realizada por el docente-investigador a través de un análisis, que emana del paradigma de la didáctica profesional (Pastré, 2011). Explora los homomorfismos (Vergnaud, 1994) entre la actividad del docente como director de una memoria de Máster y este tipo de actividad menos conocida. Se plantea entonces si los conceptos en acto (Vergnaud, 1999) construidos por el actor para poder superar la primera situación son transferibles hacia el segundo marco. En ambos casos ¿se trata de la misma situación, ampliada? En cuyo caso ¿se trataría del acompañamiento de realización de un trabajo universitario?

Si ambas situaciones de actividad parecen muy próximas, conviene esclarecer las diferencias, puesto que éstas son esenciales para los actores, ya que dichas diferencias les permiten conceptualizar mejor la especificidad de la actividad de acompañamiento de la $V A E$, para la cual se sienten poco preparados.

Palabras claves : Acompañamiento VAE (validación de la experiencia). Seguimiento de memoria. Didáctica profesional. Conceptualización.

Grégory Munoz, Emmanuel Sylvestre et Évelyne Soulard. Éléments de conceptualisation du travail d'enseignant chercheur : L'activité suivi de mémoire est-elle homomorphe à l'activité accompagnement de Validation des Acquis de l'Expérience? Les Sciences de l'éducation - Pour l'Ère nouvelle, vol. 46, $\mathrm{n}^{\circ}$ 2, 2013, pp. 41-59. ISSN 0755-9593. ISBN 978-2-918337-15-7. 